\title{
The site of administration influences both the type and the magnitude of the immune response induced by DNA vaccine electroporation
}

\author{
Gaëlle Vandermeulen ${ }^{\mathrm{a}}$, Kevin Vanvarenberg ${ }^{\mathrm{a}}$, Ans De Beuckelaer ${ }^{\mathrm{b}}$, Stefaan De Koker ${ }^{\mathrm{b}}$, \\ Laure Lambricht ${ }^{\mathrm{a}}$, Catherine Uyttenhove ${ }^{\mathrm{c}}$, Anca Reschner ${ }^{\mathrm{d}}$, Alain Vanderplasschen ${ }^{\mathrm{d}}$, \\ Johan Grooten ${ }^{\mathrm{b}}$, Véronique Préat ${ }^{\mathrm{a}, *}$ \\ a Université catholique de Louvain, Louvain Drug Research Institute, Advanced Drug Delivery and Biomaterials, Brussels, Belgium \\ b Department of Biomedical Molecular Biology, Ghent University, Ghent, Belgium \\ ${ }^{\mathrm{c}}$ Université catholique de Louvain, Ludwig Institute for Cancer Research, Brussels Branch and de Duve Institute, Brussels, Belgium \\ d Immunology-Vaccinology, Department of Infectious and Parasitic Diseases, Fundamental and Applied Research for Animals E' Health (FARAH), Faculty of \\ Veterinary Medicine, University of Liège, Liège, Belgium
}

\section{A R T I C L E I N F O}

\section{Article history:}

Received 14 November 2014

Received in revised form 10 March 2015

Accepted 4 May 2015

Available online 14 May 2015

\section{Keywords:}

DNA vaccine

Electroporation

Delivery site

Tumor vaccine

HIV vaccine

\begin{abstract}
A B S T R A C T
We investigated the influence of the site of administration of DNA vaccine on the induced immune response. DNA vaccines were administered by electroporation at three different sites: tibial cranial muscle, abdominal skin and ear pinna. Aiming to draw general conclusions about DNA vaccine delivery, we successively used several plasmids encoding either luciferase and ovalbumin as models or gp 160 and P1A as vaccines against HIV and P815 mastocytoma, respectively. Low levels and duration of luciferase transgene expression were observed after electroporation of the abdominal skin, partly explaining its lower immunogenic performance as compared to the other sites of administration. Analyses of OT-I CD8+ and OT-II CD4+ T cell responses highlighted the differential impact of the delivery site on the elicited immune response. Muscle electroporation induced the strongest humoral immune response and both muscle and ear pinna sites induced cellular immunity against gp160. Ear pinna delivery generated the highest level of CTL responses against P1A but electroporation of muscle and ear pinna were equally efficient in delaying P815 growth and improving mice survival. The present study demonstrated that the site of administration is a key factor to be tested in the development of DNA vaccine.
\end{abstract}

(c) 2015 Elsevier Ltd. All rights reserved.

\section{Introduction}

DNA vaccines are an attractive strategy to induce immune memory. An obvious advantage over protein-based vaccines is that the same methods of production, purification, quality control and preservation can be used for all vaccines [1]. In addition, the presence of immunostimulatory motifs such as CpG that are capable to activate the innate immune system endow DNA vaccine with intrinsic adjuvant properties while transfection of both antigen presenting cells (APCs) and surrounding structural cells allows the generation of humoral, cellular and CTL immune responses [2]. The efficiency of DNA vaccines has already been documented in a wide

\footnotetext{
* Corresponding author at: Université catholique de Louvain, Louvain Drug Research Institute, Avenue Mounier 73, bte B1.73.12, 1200 Brussels, Belgium. Tel.: +32 2764 7309; fax: +322 7647398 .

E-mail address: veronique.preat@uclouvain.be (V. Préat).
}

range of preclinical models and several clinical trials are currently ongoing [3].

The delivery method of DNA vaccine has often been described as a key point to achieve high level of transfection in vivo. Electroporation is a non-viral delivery method based on membrane destabilization and DNA electrophoresis, mediated by electric pulses. Efficient delivery of plasmid into muscle, skin, tumor and other tissues using in vivo electroporation has thus been demonstrated [4-10].

Skin is an attractive site for DNA vaccine administration, rich in APCs [11]. Moreover, intradermal injection can be easily performed, simple electrode devices such as plate electrodes or less invasive microneedle electrodes can be applied [12]. The skin of the ear pinna was described as a particularly effective site leading to CTL response and protective tumor immunity [13]. Yet, also muscle has been used frequently as target tissue for the administration of DNA vaccines. Muscle is easily accessible by injection through the skin and requires lower doses of DNA as compared to the other tissues as a consequence of its high transfection efficiency. Although 
low in APCs, it is generally admitted that the electric pulses promote the recruitment of immune cells and hereby initiate the cascade of events leading to immune reactivity [14].

In previous work, we and others addressed the question of the optimal administration site for the electroporation of plasmid vaccines by quantifying the expression levels and immune reactivity against luciferase $[6,15]$ and $\beta$-galactosidase reporter proteins [16]. However, the influence of the delivery site on the efficiency of DNA vaccines directed against disease-related antigens as well as the underlying immunological mechanisms remains to be fully investigated. The present work aimed to evaluate muscle, abdominal skin and ear pinna sites in two relevant preclinical models, namely the gp160 envelope HIV vaccine and the mouse P1A tumor antigen vaccine. Besides an analysis of the strength and nature of the elicited immune responses, also the impact of the site of administration on the level of transgene expression and on the proliferation of OT-I CD8+ and OT-II CD4+ T cells was studied.

\section{Materials and methods}

\subsection{Plasmids}

pVAX2 was obtained by replacing the cytomegalovirus promoter of pVAX1 (Invitrogen) by that of pCMV- $\beta$ (Clontech). pVAX2-LUC encodes luciferase. The codon optimized ovalbumin sequence was ordered from GeneArt and subcloned into pVAX2 to construct pVAX2-OVA. pcDNA3.1(-)-96ZM-gp160CD5 (gp160) encodes the gp160 HIV type-1 (HIV-1) envelope glycoprotein. pMDLg/pRRE (gag) was purchased from Addgene (Fargo, ND). pVAX2-P1A encoding the full-length P1A protein was previously constructed [17]. Plasmids were prepared using EndoFree Plasmid Mega or Giga Kit (Qiagen, Venlo, Netherlands). Optical density at $260 \mathrm{~nm}$ was used to determine DNA concentration. Plasmid quality was assessed by the $260 \mathrm{~nm} / 280 \mathrm{~nm}$ ratio and agarose gel electrophoresis. Plasmids diluted in PBS were stored at $-20^{\circ} \mathrm{C}$.

\subsection{Animals}

Balb/c, C57BL/6 and DBA/2 female mice were obtained from Harlan Nederland or Janvier France. Mice were between 6 and 8 -week-old at the beginning of the experiments. They were anesthetized by intraperitoneal injection of ketamine (Anesketin, euroVet, Belgium) and xylazine (Sigma, Belgium). Before electroporation, mice were shaved using a rodent shaver (Aesculap Exacta shaver, AgnTho's, Sweden). All experimental protocols in mice were approved by the Ethical Committee for Animal Care and Use of the Medical Sector of the Université catholique de Louvain.

\subsection{Plasmid injection and electroporation}

Injection and electroporation procedures of skin, muscle and ear pinna were previously described [6]. Plasmid doses and electroporation settings are summarized in Table 1. Briefly, plasmids were first injected using an insulin syringe. For skin and ear vaccination, we injected either $15 \mu \mathrm{l}$ into the dermis at two different sites or $30 \mu \mathrm{l}$ into the external side of the right ear pinna, respectively. $2 \mathrm{~mm}$ spaced electrodes were applied to deliver a short high voltage pulse (HV, $700 \mathrm{~V} / \mathrm{cm} 100 \mu \mathrm{s})$ immediately followed by a low voltage pulse ( $\mathrm{LV}, 200 \mathrm{~V} / \mathrm{cm} 400 \mathrm{~ms})$ using a Cliniporator system (Cliniporator, IGEA, Italy). For muscle administration, we injected $30 \mu$ into the left tibial cranial muscle, and placed the leg between $4 \mathrm{~mm}$ spaced electrodes to deliver eight square-wave electric pulses $(200 \mathrm{~V} / \mathrm{cm} 20 \mathrm{~ms} 2 \mathrm{~Hz}$ ). For the study of the OT-I and OT-II proliferation, two sites per mouse were treated: both tibial cranial muscles, left and right lower abdominal quadrants or both ears. For all experiments, conductive gel was used to ensure electrical contact with the skin (Aquasonic 100, Parker, USA).
Table 1

Electroporation settings, doses and summary of the influence of the delivery site for DNA vaccine electroporation.

\begin{tabular}{|c|c|c|c|}
\hline & Skin & Ear pinna & Muscle \\
\hline \multicolumn{4}{|l|}{ Electroporation settings } \\
\hline Electrodes & \multicolumn{2}{|c|}{$2 \mathrm{~mm}$} & \multirow{3}{*}{$\begin{array}{l}4 \mathrm{~mm} \\
/\end{array}$} \\
\hline \multirow[t]{2}{*}{$\mathrm{HV}$} & \multirow{2}{*}{\multicolumn{2}{|c|}{$\begin{array}{c}1 \text { pulse } \\
700 \mathrm{~V} / \mathrm{cm} 100 \mu \mathrm{s}\end{array}$}} & \\
\hline & & & \\
\hline \multirow[t]{3}{*}{ LV } & \multicolumn{2}{|c|}{1 pulse } & \multirow{3}{*}{$\begin{array}{l}8 \text { pulses } \\
200 \mathrm{~V} / \mathrm{cm} \\
20 \mathrm{~ms} 2 \mathrm{~Hz}\end{array}$} \\
\hline & & $/ \mathrm{cm}$ & \\
\hline & & $\mathrm{ms}$ & \\
\hline \multicolumn{4}{|l|}{ Luciferase expression } \\
\hline \multicolumn{4}{|l|}{$50 \mu g$ pVAX2-LUC } \\
\hline Magnitude & + & ++ & +++ \\
\hline Duration & + & ++ & ++ \\
\hline \multicolumn{4}{|l|}{ OT-I/OT-II proliferation } \\
\hline \multicolumn{4}{|c|}{ Two injections for a total dose of $5 \mu \mathrm{g} p V A X 2-O V A$} \\
\hline CD8+ T cells (OT-I) & + & +++ & - \\
\hline CD4+ T cells (OT-II) & - & ++ & + \\
\hline \multicolumn{4}{|l|}{ gp160 HIV } \\
\hline \multicolumn{4}{|c|}{$25 \mu \mathrm{g}$ of gp 160 and $27.6 \mu \mathrm{g}$ of gag (4.7 pmol of each plasmid) } \\
\hline Total IgG titers & - & + & ++ \\
\hline $\operatorname{IgG1}$ & & + & ++ \\
\hline $\operatorname{IgG} 2 \mathrm{a}$ & & + & + \\
\hline$\gamma$-IFN & - & ++ & + \\
\hline IL-2 & + & ++ & ++ \\
\hline IL-4 & - & + & - \\
\hline IL-10 & - & - & - \\
\hline \multicolumn{4}{|l|}{ P815 tumor } \\
\hline \multicolumn{4}{|l|}{$50 \mu \mathrm{g}$ of $p V A X 2-P 1 A$} \\
\hline CTL response & - & ++ & + \\
\hline Efficacy against challenge & - & + & + \\
\hline
\end{tabular}

\subsection{Luciferase expression}

Balb/c mice were injected with $50 \mu \mathrm{g}$ of pVAX2-LUC and electroporated. To follow the luciferase expression, mice were injected i.p. with luciferin $(150 \mathrm{mg} / \mathrm{kg}$ ) in $100 \mu$ l of PBS. Optical images were acquired at several time points using an IVIS Spectrum system (Xenogen Corporation, USA).

\subsection{OT-I and OT-II proliferation}

T cells were isolated from spleen and lymph nodes of transgenic OT-I and OT-II mice using CD8+ and CD4+ T cell isolation kit II mouse (Miltenyi Biotec, The Netherlands). Subsequently the T cells were labeled with CFSE (carboxyfluorescein diacetate succinimidyl ester) by incubating $50 \times 10^{6} \mathrm{cell} / \mathrm{ml}$ with $5 \mu \mathrm{M}$ CFSE for $10 \mathrm{~min}$ at $37^{\circ} \mathrm{C}$. The reaction was blocked by adding ice-cold PBS (Lonza, Belgium $)+10 \%$ serum. $2 \times 10^{6}$ OT-I or OT-II cells were injected into the tail vein of C57BL/ 6 mice. They were treated 2 days later by pVAX2-OVA injection and electroporation. For each site of administration, two injections were performed for a total dose of $5 \mu \mathrm{g}$ of plasmid. Mice were sacrificed 4 days later to collect the draining lymph nodes for single cell suspension preparation. Flow cytometric measurement was performed on a triple-laser (B-V-R) LSR-II with FACSDiva software (Becton Dickinson, Belgium). Analysis was done with FlowJo Software (Treestar, USA). Cells were stained with aqua live dead (Invitrogen, Belgium), $\alpha$-CD16/CD32, CD19 APC-Cy7, CD8 PerCP, CD3 V450 (all BD Biosciences), dextramer SIINFEKL H2kb PE (Immudex, Denmark).

\section{6. gp160 HIV model}

BALB/c mice were immunized by co-injection of $25 \mu \mathrm{g}$ of gp160 plasmid and $27.6 \mu \mathrm{g}$ of gag plasmid, followed by electroporation [18]. They received one priming and two boosts, 2 and 4 weeks later. Two weeks after delivery of the last boost, blood 

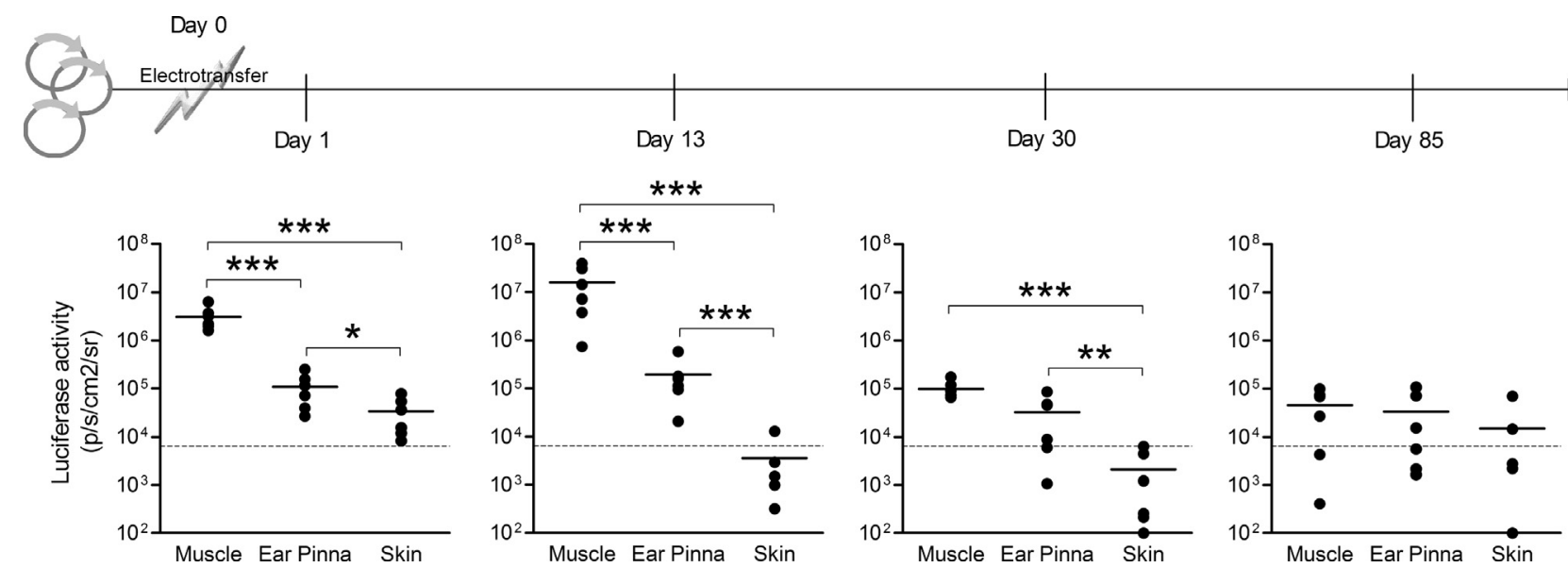

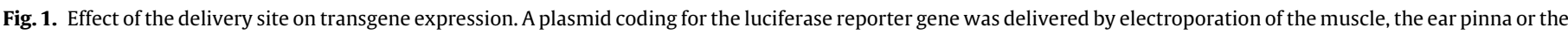

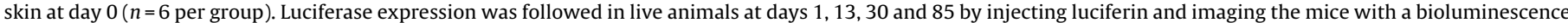
camera. Statistical analysis: one-way ANOVA with Tukey post-test. ${ }^{*} p$ value $<0.05,{ }^{* *} p$ value $<0.01,{ }^{* * *} p$ value $<0.001$.

samples were collected and an ELISA assay was performed. 96well plates were coated at $4{ }^{\circ} \mathrm{C}$ overnight with $5 \mu \mathrm{g} / \mathrm{ml}$ of HIV gp160 protein (Fitzgerald, USA) diluted in PBS. After washing with PBS/Tween 20 0.05\%, plates were blocked with 5\% dry milk in PBS for $2 \mathrm{~h}$ at $37^{\circ} \mathrm{C}$. Plates were then washed and incubated for $2 \mathrm{~h}$ at $37^{\circ} \mathrm{C}$ with serial 2 -fold dilutions of sera samples diluted in $1 \%$ dry milk in PBS. After washing, peroxidase-labeled LO-MGCOC2 (IMEX, UCL, Brussels, Belgium) and TMB substrate revelation (Calbiochem, USA) were used to determine total immunoglobulin titers, defined as the dilution factor giving an optical density at $450 \mathrm{~nm}$ equal to the limit of quantification (LOQ mean blank value plus $10 \mathrm{SDs}$ ). Isotypes of anti-gp160 antibodies were determined using appropriate secondary antibodies (LO-MG1-13, LO-MG2A9 ). Mice were sacrificed 18 days after the second boost and their spleens were collected aseptically. Red blood cells were removed with ACK lysis buffer (Lonza, USA) and splenocytes washed with PBS, counted and adjusted to a concentration of $10^{6}$ cells/well. They were cultured in 96-well tissue culture plates (Becton Dickinson, Belgium) in RPMI 1640 medium supplemented with $10 \%$ FBS, $1 \%$ penicillin/streptomycin, $1 \%$ sodium pyruvate, $5 \times 10^{-5} \mathrm{M}$ 2-mercapto-ethanol and 10\% MEM (Gibco, Belgium) as previously described [6]. Cells were restimulated by the addition of $100 \mu \mathrm{l}$ of supernatant from gp160 plasmid-transfected 293T cells, $100 \mu \mathrm{l}$ concanavalin A $(5 \mu \mathrm{g} / \mathrm{ml})$ as a positive control or $100 \mu \mathrm{l}$ of culture medium as a negative control. After $48 \mathrm{~h}$ of incubation $\left(37^{\circ} \mathrm{C}, 5 \%\right.$ $\mathrm{CO}_{2}$ ), supernatants were collected and assayed for IFN- $\gamma$, IL-2, IL4 and IL-10 (DuoSet ELISA development kits, R\&D Systems Europe Ltd, UK).

\subsection{P815 tumor model}

DBA/2 mice were immunized by injection of $50 \mu \mathrm{g}$ of pVAXP1A and electroporation. Two boosts were similarly applied 2 and 4 weeks after the priming. As a positive control, mice were immunized by two intra-peritoneal injections of $10^{6}$ L1210.P1A.B7.1 living cells [19]. After immunization, mice were either used to analyze the CTL response or challenged [17]. Briefly, the peripheral blood lymphocytes were isolated and restimulated in vitro with L1210.P1A.B.7.1 cells. Lytic activity was measured in a chromium release assay $[20,21]$. Specific lytic unit (LU) was defined as the number of cells that lyse $50 \%$ of $10^{4}$ target cells in $4 \mathrm{~h}$. As a challenge, $10^{6}$ P1A-expressing P815B cells were injected subcutaneously into the flank and the tumor size (i.e. length $\times$ width $\times$ height, in $\mathrm{mm}^{3}$ ) was measured with an electronic digital caliper. Mice were sacrificed when the volume of the tumor became larger than $1500 \mathrm{~mm}^{3}$ or when they were in poor condition.

\subsection{Statistical analyses}

t-Test, one-way ANOVA with Tukey post-test and two-way ANOVA with Bonferroni post-test were used. Statistical analyses were performed using the software GraphPad Prism 5 for Windows.

\section{Results}

\subsection{The delivery site influenced the magnitude and the duration of luciferase expression after electroporation}

Luciferase reporter gene was used in order to study the impact of the delivery site on the level of DNA vaccine expression (Fig. 1). After 1 day, gene expression was observed in all mice. Those treated by muscle electroporation showed the highest level of expression which was 28 -fold and 93-fold compared to those obtained after electroporation of ear pinna and skin, respectively. After 13 days, the expression levels dropped to below the limit of quantification for mice treated into the abdominal skin. After 1 month, expression was still detectable in the mice treated into the muscle or the ear pinna. Finally, 85 days after treatment, low levels of expression were measured whatever the DNA administration site.

\subsection{T cell response was dependent on the delivery site}

To investigate the impact of the delivery site on the induction of T cell responses in vivo, CFSE-labeled OT-I CD8+ or OT-II CD4+ T cells were transferred into mice that were then treated by pVAX2-OVA electroporation. The draining lymph nodes were collected and OT-I and OT-II proliferation assessed by flow cytometry. After electroporation of the muscles with $5 \mu \mathrm{g}$ of plasmid, OT-I cells remained undivided while a slight proliferation was observed for OT-II cells (Fig. 2A and B, respectively). After electroporation of the abdominal skin, OT-I cells divided while OT-II cells failed to proliferate. Finally, the stronger proliferation was observed for both OT-I and OT-II cells after electroporation of the ear pinnas but the division of OT-I cells appeared to be higher than OT-II cells with $7.5 \%$ and $47.5 \%$ of undivided cells, respectively. Together, these results provide evidence that the delivery site has a strong influence on CD8+ and CD4+ T cell responses in vivo. 
A

Divided OT-I cells

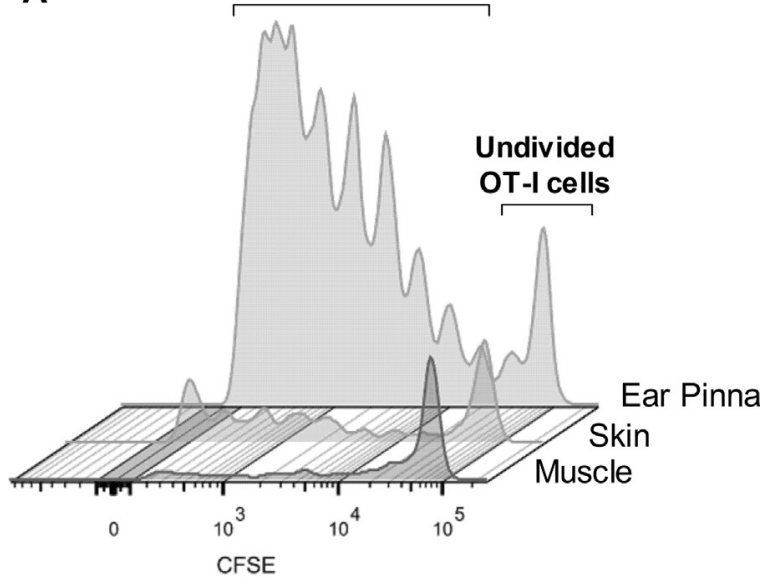

B

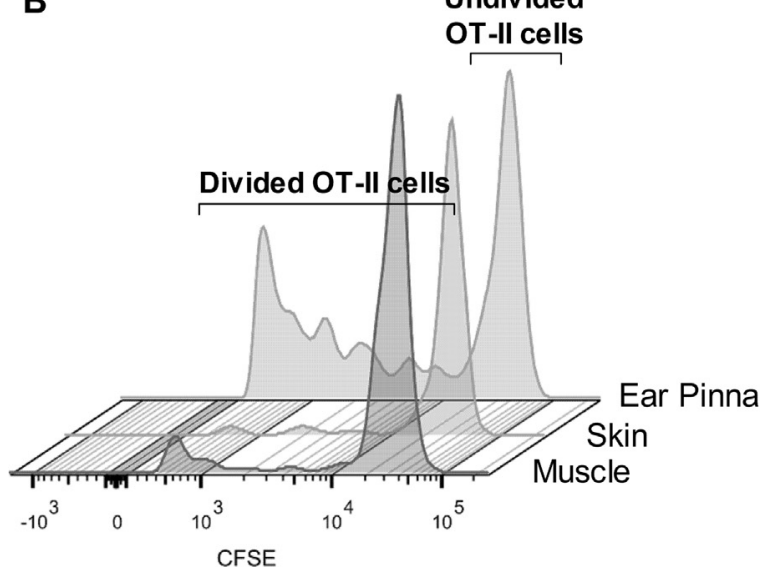

Fig. 2. Flow cytometry histograms of OT-I (panel A) and OT-II (panel B) in vivo proliferation. $5 \mu \mathrm{g}$ of the ovalbumin plasmid was delivered by electroporation into the muscle, the abdominal skin or the ear pinna at day 0 ( $n=3$ per group). T cells proliferation was assessed 4 days later.

\subsection{Muscle electroporation elicited the strongest humoral response against gp160}

Strong immune responses against gp160 were obtained in mice when a plasmid encoding the gp160 HIV-1 envelope was combined with a plasmid coding for the gag virion structural protein [18]. Here, after one priming and two boosts delivered every 2 weeks, mice that were vaccinated intramuscularly showed the highest anti-gp160 IgG levels. Levels of IgG against gp160 were also significantly increased when the DNA vaccine was delivered intradermally into the ear pinna but remained very low and similar to the untreated control mice when delivered into the dermis of the abdomen (Fig. 3A). The anti-gp160 IgG titers were 1.6 times and 16 times higher after immunization into the muscle as compared to the ear pinna and abdominal skin, respectively (Fig. 3B). To investigate if the delivery site could also influenced the bias of the immune response, we measured the IgG1 and IgG2a specific titers. Both isotypes were detected after immunization by electroporation into the muscle and the ear pinna, indicating that both Th1 and Th2 arms of the immune system were stimulated. However, whereas these two administration sites resulted in similar IgG2a titers, IgG1 titers were significantly increased after intramuscular delivery (Fig. 1C). The resulting IgG1/IgG2a ratios were 1.1 and 5.1 for ear pinna and muscle delivery sites respectively, suggesting
A

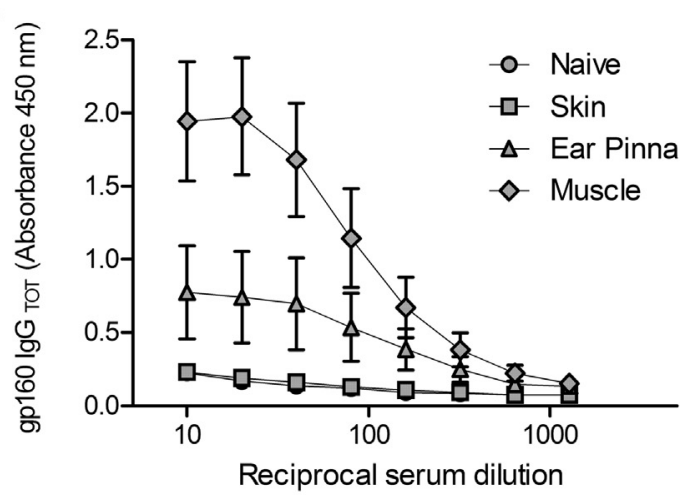

B

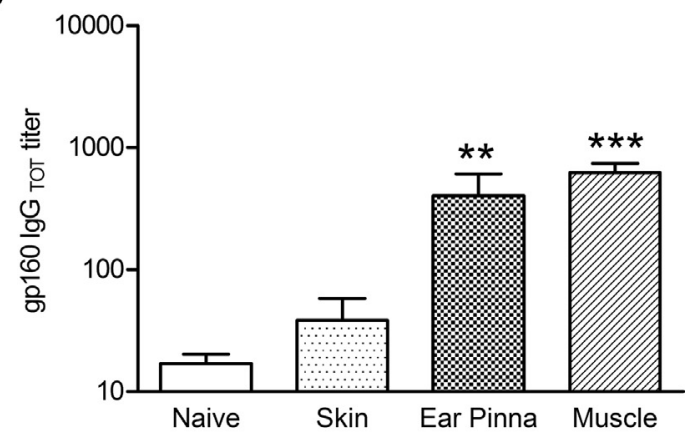

C

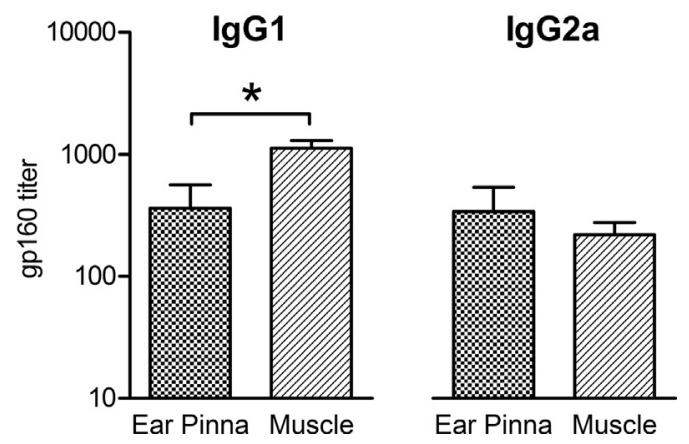

Fig. 3. Influence of the delivery site on the humoral immune response directed to gp160 measured 2 weeks after the end of the immunization. Priming and two boosts were delivered into the muscle $(n=6)$, the abdominal skin $(n=5)$ or the ear pinna $(n=6)$ by gp160 and gag plasmids injection and electroporation. Naive mice remained untreated $(n=5)$. Panel A: total anti-gp160 IgG as a function of sera dilution measured by ELISA. Panel B: total anti-gp160 IgG titers. Panel C: anti-gp160 titers for the IgG1 and IgG2a isotypes for mice immunized by the ear pinna or the muscle site. Graphs represent the mean values ( \pm SEM). Statistical analysis: one-way ANOVA with Tukey post-test as compared to naive (panel B) and $t$-test (panel C) ${ }^{*} p$ value $<0.05,{ }^{* *} p$ value $<0.01,{ }^{* * *} p$ value $<0.001$

the occurrence of a stronger humoral response against gp160 after muscle electroporation.

\subsection{The site of electroporation influenced cytokines secretion by gp160 restimulated splenocytes}

To further study the role of the administration site on the generation of immune response, secretion of various cytokines was measured after restimulation of splenocytes collected from animals immunized by gp160 and gag plasmids injection and electroporation (Fig. 4). IFN- $\gamma$ was significantly induced when the ear pinna or the muscle site were used but remained low when the abdominal skin site was used for immunization. A significant increase of 




$\mathrm{IFN}-\gamma(\mathrm{ng} / \mathrm{ml})$

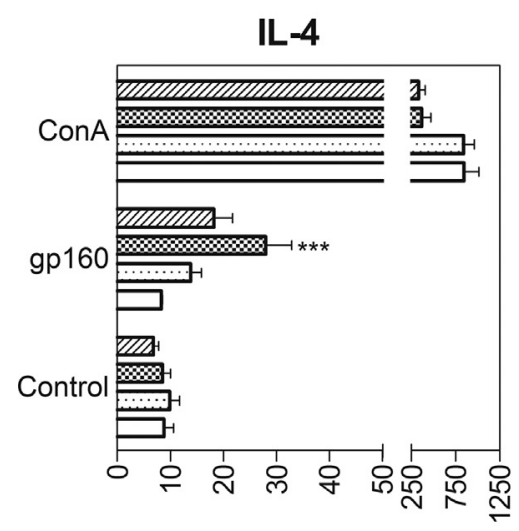

IL-4 (pg/ml)
IL-2

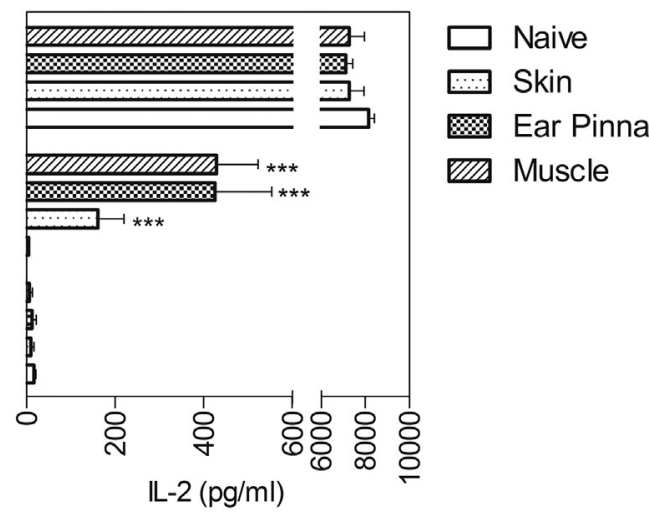

IL-10

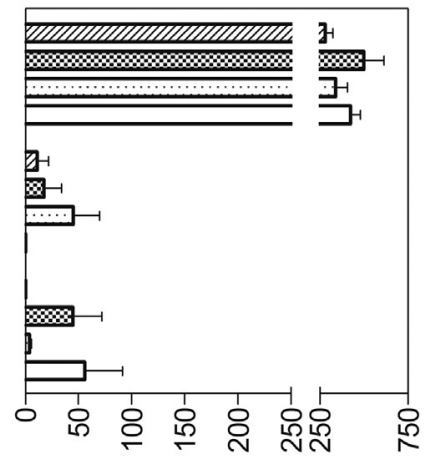

$\mathrm{IL}-10(\mathrm{pg} / \mathrm{ml})$

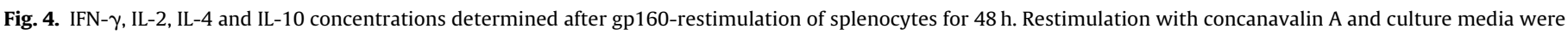

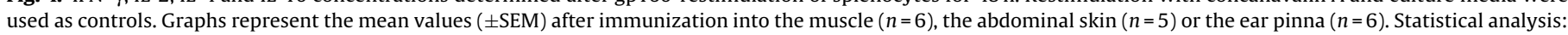
one-way ANOVA with Tukey post-test. ${ }^{*} p$ value $<0.05,{ }^{* *} p$ value $<0.01,{ }^{* * *} p$ value $<0.001$ as compared to naive (restimulated with gp160).

IL-2 was observed for all the DNA vaccine administration sites but higher levels were obtained after immunization through ear pinna or muscle. Secretion of IL-4 was higher for mice immunized by electroporation of ear pinna. No significant increase of IL-10 was observed whatever the delivery site.

\subsection{Ear pinna was the optimal site to generate a CTL response}

A DNA vaccine encoding the mouse P1A tumor antigen was previously reported to delay the outgrowth of P815 mastocytoma, a preclinical model for human tumors expressing MAGE tumor antigens [17]. The CTL response generated by the vaccine was evaluated by a chromium release assay after in vitro restimulation of immune spleen cells. No CTL reactivity was detectable when mice were immunized by the abdominal skin site. In contrast herewith, a clear anti-P1A response was observed when the vaccine was delivered into the muscle and was at his strongest when the DNA vaccine was administered through ear pinna (Fig. 5A).

\subsection{Muscle and ear pinna electroporation were equally efficient in delaying P815 tumor growth and improving mouse survival}

Vaccinated mice were challenged with P1A-expressing P815B cells. Mice immunized with L1210.P1A.B7.1 as a positive control were efficiently protected against the tumor cells. Electroporation of the P1A vaccine into the abdominal skin failed to induce rejection of P815B cells or even to delay tumor growth (Fig. 5B and C). Interestingly, electroporation of the same vaccine into the muscle and the ear pinna significantly impaired P815 tumor growth and promoted a long term survival of approximately $40 \%$ of the treated mice, equal to mice vaccinated with the reference vaccine, L1210.P1A.B7.1.

\section{Discussion}

This study reveals a strong influence of the delivery site on the type and magnitude of the immune responses elicited by DNA vaccine electroporation as summarized in Table 1.

The skin is quite often selected for the immunization studies due to its easy accessibility. However, electroporation of the abdominal skin resulted in very low immune responses against both gp160 HIV and P815 tumor vaccines. This could be partly explained by the low level of protein expression we observed after intradermal electroporation. Moreover, electroporation of the skin completely failed to induce CD4+ T cells proliferation in a mouse model using TCR transgenic OT-II T cells. Although some CD8 $+\mathrm{T}$ cell reactivity was observed in the same experimental setup as well as secretion of IL-2 after immunization with gp160 DNA vaccine, the cellular immune response elicited was not sufficient to generate a protective immune response against the P815 tumor.

Interestingly, the skin of the ear pinna showed particular features that drastically changed the outcome of DNA vaccine electroporation. First, a humoral response was noticeable after electroporation of the gp160 HIV DNA vaccine, with the presence of both IgG1 and IgG2a isotypes. In the same model, IFN- $\gamma$, IL-2 and IL-4 were secreted after ex vivo stimulation of splenocytes with the gp160 envelope protein. Immunization through the ear pinna site also resulted in high CTL response and allowed a significant tumor growth delay and a long term survival of vaccinated mice after 


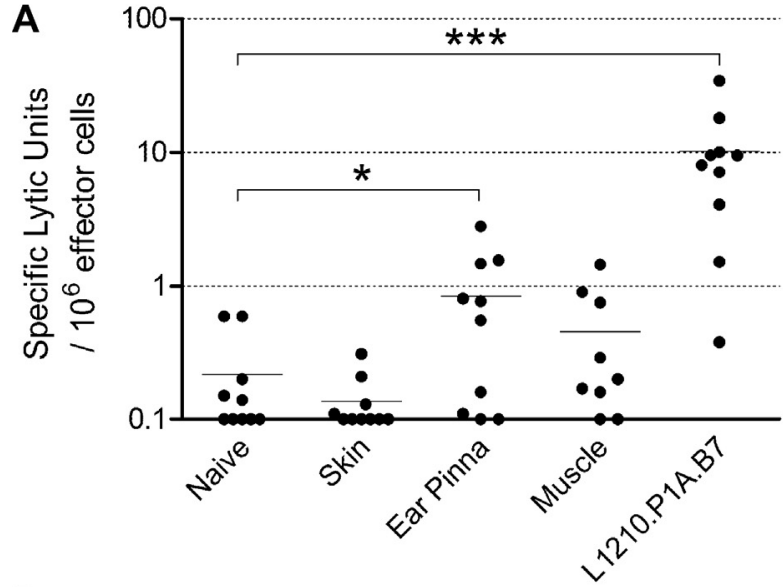

B



Day after challenge

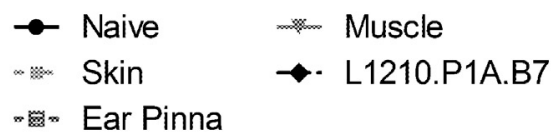

C



Day after challenge

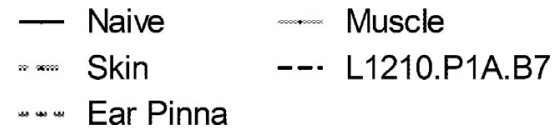

Fig. 5. Influence of the delivery site on the immune response against the tumor antigen P1A. The P1A DNA vaccine was delivered by electroporation of the muscle $(n=10)$, the abdominal skin $(n=10)$ or the ear pinna $(n=9)$. Mice immunized by i.p. injections of L1210.P1A.B7.1 cells $(n=10)$ and naive mice $(n=10)$ were used as controls. Panel A: P1A-specific CTL responses after immunization. Panel B: evolution of tumor volumes after challenge with $10^{6}$ P1A-expressing P815B cells. Graph represents the mean tumor size $( \pm$ SEM). Panel C: survival curves after P815 challenge. Statistical analysis: one-way ANOVA with Tukey post-test (panel A) and two-way ANOVA with Bonferroni post-test (panel B). ${ }^{*} p$ value $<0.05,{ }^{* *} p$ value $<0.01,{ }^{* * *} p$ value $<0.001$ as compared to naive.
P815 challenge. The strong and prolonged protein expression we observed using the ear pinna as compared to the abdominal skin is certainly one of the key elements to explain the strong immunizing outcome of this particular site.

Electroporation of the tibial cranial muscle allowed the generation of high anti-gp160 antibody titers with a Th2 bias while only a slight CD4+ T cells response was observed for the ovalbumin model vaccine. The P1A vaccine delivered into the muscle reduced tumor growth suggesting that the muscle was also a valuable site for generating a cellular immune response. Interestingly, it appeared that the differences observed in terms of immune response cannot be solely attributed to the level of antigen expression. Indeed, if muscle electroporation generated very high transgene expression as compared to electroporation of the ear pinna site, this latest demonstrated very good immunogenic performance throughout the study.

In conclusion, the present work underlines the need for a conscious, careful and rational choice of the delivery site for the electroporation of DNA vaccines. Four different models have been used in this study which highlighted general trends about the influence of the delivery site on the immune outcome and could therefore guide the choice of the electroporation site for preclinical evaluation of DNA vaccine candidates. The skin of the ear pinna could be selected as the site of choice when a pronounced cellular immune response is required such as in the case of cancer vaccines or vaccines against intracellular pathogens. The intramuscular site should be preferred when a humoral response is needed after electroporation of the DNA vaccine or when both the humoral and the cellular arms of the immune defense must be activated. In addition to the site of delivery, several parameters should be taken into account for the development of DNA vaccines such as the plasmid itself (backbone, gene promoter, sequence optimization, secretion signal, etc.) or the electrode design and a better understanding of these factors will be critical for the future of DNA vaccine strategies.

\section{Acknowledgments}

We are grateful to Bernard Ucakar, Nathalie Lecouturier and Dominique Donckers for their technical help. François Hippeau is acknowledged for his contribution to this work and Cédric Szpirer (Delphi Genetics) for helpful discussions. The authors also thank Pascal Bigey and Daniel Scherman (University Paris V) for the gift of pVAX2 and pVAX2-LUC plasmids and George Dickson (Royal Holloway University of London) for the gp160 plasmid. This work was supported by grants from the Belgian Fonds National de la Recherche Scientifique (F.R.S.-FNRS) and from the Walloon region through DNAVAC, a certified BioWin project (DG06). Gaëlle Vandermeulen is a postdoctoral researcher of the Fonds de la Recherche Scientifique-FNRS.

Conflict of interest statement: The authors declare that they have no conflict of interest.

\section{References}

[1] Carnes AE, Williams JA. Plasmid fermentation process for DNA immunization applications. Methods Mol Biol 2014;1143:197-217.

[2] Tudor D, Dubuquoy C, Gaboriau V, Lefevre F, Charley B, Riffault S. TLR9 pathway is involved in adjuvant effects of plasmid DNA-based vaccines. Vaccine 2005;23(January (10)):1258-64

[3] www.clinicaltrials.gov, 2014

[4] Bettan M, Emmanuel F, Darteil R, Caillaud JM, Soubrier F, Delaere P, et al. Highlevel protein secretion into blood circulation after electric pulse-mediated gene transfer into skeletal muscle. Mol Ther 2000;2(September (3)):204-10.

[5] Mir LM, Bureau MF, Gehl J, Rangara R, Rouy D, Caillaud JM, et al. High-efficiency gene transfer into skeletal muscle mediated by electric pulses. Proc Natl Acad Sci U S A 1999;96(April (8)):4262-7.

[6] Vandermeulen G, Staes E, Vanderhaeghen ML, Bureau MF, Scherman D, Preat V. Optimisation of intradermal DNA electrotransfer for immunisation. J Control Release 2007;124(December (1-2)):81-7. 
[7] Gothelf A, Hojman P, Gehl J. Therapeutic levels of erythropoietin (EPO) achieved after gene electrotransfer to skin in mice. Gene Ther 2010;17(September (9)):1077-84.

[8] Steinstraesser L, Lam MC, Jacobsen F, Porporato PE, Chereddy KK, Becerikli M, et al. Skin electroporation of a plasmid encoding hCAP-18/LL-37 host defense peptide promotes wound healing. Mol Ther 2014;22(April (4)):734-42.

[9] Lucas ML, Heller L, Coppola D, Heller R. IL-12 plasmid delivery by in vivo electroporation for the successful treatment of established subcutaneous B16.F10 melanoma. Mol Ther 2002;5(June (6)):668-75.

[10] Daugimont L, Vandermeulen G, Defresne F, Bouzin C, Mir LM, Bouquet C, et al. Antitumoral and antimetastatic effect of antiangiogenic plasmids in B16 melanoma: higher efficiency of the recombinant disintegrin domain of ADAM 15. Eur J Pharm Biopharm 2011;78(August (3)):314-9.

[11] Cui Z, Dierling A, Foldvari M. Non-invasive immunization on the skin using DNA vaccine. Curr Drug Deliv 2006;3(January (1)):29-35

[12] Daugimont L, Baron N, Vandermeulen G, Pavselj N, Miklavcic D, Jullien MC, et al. Hollow microneedle arrays for intradermal drug delivery and DNA electroporation. J Membr Biol 2010;236(July (1)):117-25.

[13] Forg P, von HP, Dalemans W, Schirrmacher V. Superiority of the ear pinna over muscle tissue as site for DNA vaccination. Gene Ther 1998;5(June (6)):789-97.

[14] Gothelf A, Gehl J. What you always needed to know about electroporation based DNA vaccines. Hum Vaccin Immunother 2012;8(November (11)):1694-702.

[15] Petkov SP, Heuts F, Krotova OA, Kilpelainen A, Engstrom G, Starodubova ES, et al. Evaluation of immunogen delivery by DNA immunization using non-invasive bioluminescence imaging. Hum Vaccin Immunother 2013;9(October (10)):2228-36.

[16] Ni J, Nolte B, Vandermeulen G, Preat V, Scherman D, Schirrmacher V, et al. Ear pinna: a privileged DNA electroporation site for inducing strong Th1 immune responses. Open Cancer Immunol J 2009;2(September):18-30.

[17] Vandermeulen G, Uyttenhove C, De PE, Van den Eynde BJ, Preat V. Intramuscular electroporation of a P1A-encoding plasmid vaccine delays P815 mastocytoma growth. Bioelectrochemistry 2014;100(December):112-8.

[18] Vandermeulen G, Athanasopoulos T, Trundley A, Foster K, Preat V, YanezMunoz RJ, et al. Highly potent delivery method of gp160 envelope vaccine combining lentivirus-like particles and DNA electrotransfer. J Control Release 2012;159(May (3)):376-83

[19] Gajewski TF, Renauld JC, Van Pel A, Boon T. Costimulation with B7-1, IL-6, and IL-12 is sufficient for primary generation of murine antitumor cytolytic T lymphocytes in vitro. J Immunol 1995;154(June (11)):5637-48.

[20] Naslund TI, Uyttenhove C, Nordstrom EK, Colau D, Warnier G, Jondal M, et al. Comparative prime-boost vaccinations using Semliki Forest virus, adenovirus, and ALVAC vectors demonstrate differences in the generation of a protective central memory CTL response against the P815 tumor. J Immunol 2007; 178(June (11)):6761-9.

[21] Uyttenhove C, Godfraind C, Lethe B, Amar-Costesec A, Renauld JC, Gajewsk $\mathrm{TF}$, et al. The expression of mouse gene P1A in testis does not prevent safe induction of cytolytic T cells against a P1A-encoded tumor antigen. Int J Cancer 1997;70(January (3)):349-56. 